\title{
Problems with immunomodulation test our clinical reasoning skills
}

\section{Sophia Samuel}

\section{Scientific reasoning is a kind of dialogue between the possible and the actual, between what might be and what is in fact the case. ${ }^{1}$}

The host immune system appears to have a fundamental role in almost all human disease. The core immune process is often articulated as the physiological mechanisms that provide both distinction and mediation between self and non-self. ${ }^{2}$ Medical interventions affecting these immune processes have reduced mortality and morbidity through a diverse range of applications including vaccination, organ transplantation, rheumatology, nephrology, transfusion and oncology.

Despite major discoveries, not all the critical pieces in the immune process have been fully uncovered. There are clues and hints related to how allergy, chronic inflammation, cancer and autoimmunity might eventuate. ${ }^{2}$ For instance, innate pattern recognition receptors on certain immune and epithelial cells trigger an inflammatory cascade in the presence of pathogens or damaged tissue. Yet, commensal bacteria, such as gut microbiota, may have the same surface receptors as the pathogens but do not trigger the cascade. ${ }^{3}$

Problems with immune modulation-due to environmental agents, internal processes and pharmacological agents - result in a range of challenging clinical presentations for the general practitioner (GP), some of which we examine in this issue of Australian Journal of General Practice.

Some are arguably straightforward, given much of the evidence and processes are clearly defined. Childhood vaccinations are a global public health achievement that has been attenuated by complacency and misinformation. ${ }^{4}$ Many patients with newly diagnosed human immunodeficiency virus can now receive vaccination when their CD4+ T cell count is known ${ }^{5}$ as part of an overall strategy that supports lifelong antiretroviral therapy.

Other clinical situations are potentially more complex, such as the patient with an autoimmune condition who now presents with an acute febrile illness. The prescribed immune-modulator or biological medication for the chronic disease acts as a suppressant or modifier of the immune response to the acute infective agent. This may be exacerbated by other factors, such as extremes of age or diabetes. All GPs are critically aware that the traditional history, examination and investigative findings in these patients may be altered and, more concerningly, quite subtle or absent.

A similar range of host factors seems to shape the clinical presentation of Mycobacterium tuberculosis infection. Patients with asymptomatic, non-contagious latent tuberculosis may be under-recognised in Australia. There is now evidence that a subsection of this group may benefit from GP-led isoniazid therapy. ${ }^{6}$

International travel is an economic necessity, an aspect of family reunification or an enjoyable leisure activity for many of our patients. Travel-related health advice is often a negotiation between multiple realised and potential health risks. The added dimension of travel while immunosuppressed requires special consideration when prescribing travel medications and advice. ${ }^{7}$

Clinical indications for immunemodulating medications can be expected to expand. GPs and tertiary health teams will need to work closely to facilitate the consequent increasingly intricate care. Prolonged use of immunemodulating medications is associated with exacerbations of metabolic disease, infections and malignancies. Impaired renal function and increased rates of cardiovascular disease and skin cancer are key reasons for increased vigilance. Case-finding strategies and aggressive medical management need to be balanced by the burden of treatment on patients. Older patients particularly face a number of unique medication-related challenges. ${ }^{8}$ GPs might be the first to suspect pharmacotoxicity or adherence problems.

Immunology is a promising field for translational research, and the astonishing advances of the past hundred years bode well for frontiers being breached in clinical advances for patients in the next few decades. An ongoing task for clinicians is to integrate this knowledge and its applied evidence base with the careful clinical reasoning necessary in every patientdoctor consultation.

\section{Author}

Sophia Samuel FRACGP, FARGP; Medical Editor at Australian Journal of General Practice and General Practitioner in Melbourne, Vic.

\section{References}

1. Medawar PB. Induction and intuition in scientific thought. London: Routledge, 1969; p. 48.

2. Hato T, Dagher PC. How the innate immune system senses and causes trouble. Clin J Am Soc Nephrol 2015;10(8):1459-69. doi: 10.2215/ CJN.04680514.

3. Chu H, Mazmanian SK. Innate immune recognition of the microbiota promotes host-microbial symbiosis. Nat Immunol 2013;14(7):668-75. doi: 10.1038/ni.2635

4. Alexander KE, Wickens M, Fletcher-Lartey SM. Measles elimination in Australia: Hard won, easily lost. Aust J Gen Pract 2020;49(3):112-14.

5. Meher-Homji Z, Giles ML. Vaccination in the person with newly diagnosed HIV. Aust J Gen Pract 2020;49(3):93-98.

6. Denholm J, Baker AM, Timlin M. Latent tuberculosis in the general practice context. Aust J Gen Pract 2020;49(3):107-10.

7. Zwar NA. Travel and immunosuppressant medication. Aust J Gen Pract 2020;49(3):88-92.

8. Ivulich S, Snell G. Long-term management of elderly patients taking immunosuppressive medications. Aust J Gen Pract 2020;49(3):100-06. 